The

Roper-Logan-Tierney

Model in Action 
The Roper-Logan-Tierney Model in Action

C. NEWTON

Peplau's Model in Action

H. S I M P S ON

Orem's Model in Action

S . CA V A N A G H

Further models in preparation

Neumann's Model in Action

Riehl's Model in Action 


\title{
The
}

\section{Roper-Logan-Tierney Model in Action}

\section{CHARLEEN NEWTON}

\section{RGN, Dip.Nursing (Lond.)}

Research \& Development Department,

Royal Hampshire County Hospital,

Winchester

\author{
Series Editor \\ BOB PRICE \\ BA, M.Sc., SRN, Cert.Ed.(Education) \\ Army Medical Services School of Nursing, \\ Woolwich
}

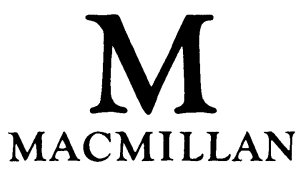


(C) Charleen Newton, 1991

All rights reserved. No reproduction, copy or transmission of this publication may be made without written permission.

No paragraph of this publication may be reproduced, copied or transmitted save with written permission or in accordance with the provisions of the Copyright, Designs and Patents Act

1988 or under the terms of any licence permitting limited copying issued by the Copyright Licensing Agency, 33-4 Alfred Place, London WC1E 7DP.

Any person who does any unauthorised act in relation to this publication may be liable to criminal prosecution and civil claims for damages.

First edition 1991

Published by

MACMILLAN EDUCATION LTD

Houndmills, Basingstoke, Hampshire RG21 2XS and London

Companies and representatives throughout the world

Designed by Claire Brodmann

Typeset by TecSet Ltd, Wallington, Surrey.

British Library Cataloguing in Publication Data

Newton, Charleen

The Roper-Logan-Tierney model in action

1. Nursing

I. Title II. Series

610.73

ISBN 978-0-333-52134-2

ISBN 978-1-349-11418-4 (eBook)

DOI 10.1007/978-1-349-11418-4 
Preface

$A$ note about language

Acknowledgements

PART I THE ROPER-LOGAN-TIERNEY MODEL TODAY

1 Models of nursing: a starting point 3

In the beginning 3

The development of the Roper-Logan-Tierney model 5

What is a nursing model?

Constructing a nursing model 7

Why do we need nursing models? 11

Summary 11

2 The model of nursing and the nursing process 13

Early development 13

Key concepts in the model $\quad 15$

3 The components of the model and the nursing process 20

Activities of living $\quad 20$

The life span $\quad 24$

The dependence-independence continuum 25

Factors influencing activities of living 25

Individuality 26

The model and the nursing process $\quad 26$

Summary $\quad 31$

PART II APPLYING THE MODEL

4 Care study: a patient undergoing major bowel surgery 35

Mrs Margaret Wells $\quad 35$

Admission $\quad 36$

$\begin{array}{ll}\text { Pre-operative care } & 40\end{array}$

Post-operative care $\quad 44$

Summary $\quad 54$

Exercises and activities $\quad 54$

5 Care study: a patient with a myocardial infarction 72

Mr James Matthews $\quad 72$

$\begin{array}{ll}\text { Admission } & 73\end{array}$

$\begin{array}{ll}\text { Nursing care } & 73\end{array}$ 
Summary

Exercises and activities $\quad 87$

6 Care study: a child needing eye surgery 99

George Lawrence $\quad 99$

$\begin{array}{lr}\text { Pre-operative care } & 100\end{array}$

Post-operative care $\quad 105$

$\begin{array}{ll}\text { Summary } & 106\end{array}$

Exercises and activities 106

7 Care study: an elderly lady 117

Mrs Annie Coleman 117

Admission $\quad 117$

Nursing care $\quad 124$

Discharge $\quad 127$

Summary $\quad 132$

Exercises and activities 133

8 Care study: a young person with diabetes 155

Ms Jody Colcross 155

Admission 156

Nursing care: activities of living 158

Nursing care: medical prescription $\quad 160$

$\begin{array}{ll}\text { Summary } & 160\end{array}$

Exercises and activities 161

PART III CRITIQUE OF THE MODEL

9 Analysis and evaluation 173

Analysis $\quad 174$

$\begin{array}{ll}\text { Evaluation } & 181\end{array}$

Evaluating the model in practice $\quad 186$

Summary 191

Appendix: Automated care planning 194

Patient care plans 196

The nursing process 196

Operating automated care planning $\quad 198$

Summary 199

Bibliography 203

$\begin{array}{ll}\text { Index } & 210\end{array}$ 
The nursing profession has become increasingly familiar with the idea of nursing models, and has, at least in part, accepted them as a means to enhance nursing care. Such models have frequently emanated from the United States of America and have, quite naturally, been framed within the idiom and culture of American nurses. The Roper-Logan-Tierney model of nursing care provides a useful exception to this rule, and for many will be a framework of care that at once seems practical, approachable and functional.

The work of Roper, Logan and Tierney is widely known, but perhaps not fully understood within their native United Kingdom. British nurses are often tempted to select the 'activities of living' component of this model and to use this as a short checklist for care-giving, ignoring the model's other dimensions. This is a surprising mistake, given that this model is widely taught in Colleges of Nursing and espoused as a model of care for wards, departments and, sometimes, whole institutions. It is particularly apt, then, that Charleen Newton's refreshing look at this British model of nursing should be one of the first volumes in the Nursing Models in Action series.

Such a review is important for four very good reasons. First, and most generally, the profession has reached a point when all models of care are receiving critical attention. The early criticism of the medical model was well founded, but now, as the profession grows, we realise that we cannot rest on our own laurels, but must examine that which replaces the medical model. Nurses are asking the pertinent question, does this model of care enhance my practice, the way I care for a patient? If so, how does it do so and does this benefit hold good with all clients in every situation?

Charleen Newton's review is also important because many nurses will already be partially utilising this model of care, and may believe, perhaps erroneously, that they have grasped all its component parts and evaluated it fairly. In my experience this is often not the case. Activities of living are understood to the extent of compiling a checklist, but perhaps little more. The model is then evaluated, compared with others and found wanting, because the overlooked elements have not been given due attention. In this text the reader will enjoy a much fuller exploration of Roper, Logan and Tierney's work. 
Thirdly, if this model is to be employed in college curricula, it is essential that it be reviewed, and applied to practical situations, by a nurse who is involved in the improved application of theory to practice. This author is ideally suited to this role, impartially examining the strengths and weaknesses of theory as she explores the best way to plan care in her own hospital setting. Fresh, researchrelated care studies are a feature of this text, and should prove invaluable to students and teachers of nursing alike.

Lastly, no model remains constant, and it will be clear from the following chapters that this one is no exception. It has developed and grown with the ongoing work of its authors and of those nurses who have sought to use this model of care. It is now appropriate to take stock of such change and - for nurses who seek to understand nursing theory - to put such work in the context of nursing culture and the current climate. The evaluation section of this text suits this purpose admirably, and will prove a welcome aid to those nurses studying at diploma, undergraduate or postgraduate level.

Reading through some of the texts on nursing models, you may be surprised to find the Roper-Logan-Tierney model unreferenced. Conversely, you may not be surprised at all, believing that this model doesn't quite fulfil the criteria of a classic nursing model. Either way, I suggest it's time to look again at all such beliefs and positions; an activity that is facilitated by this very practical book.

Bob Price 
Certain conventions have been observed in the writing of this book. First, a person receiving nursing care is usually referred to as a 'patient', but in some contexts as a 'client'; the terms are often interchangeable and their use tends to be dictated by local practice. Secondly, although people in the book are introduced with their full names and titles they are usually referred to thereafter by their first name only - this reflects the trust established in effective nursepatient relationships; each patient is understood to have consented to this use of his or her name. Thirdly, unless the context requires otherwise the nurse is referred to as 'she' and the patient as 'he'. These conventions are solely for reasons of simplicity, clarity and style. 
I should like to thank my colleagues in the Research and Development Department at the Royal Hampshire County Hospital for their support and interest; the librarian and staff in the postgraduate medical centre at the Hospital for all their help; my supervisor at Southampton University for his encouragement and also patience in waiting for me to produce my thesis; Bob Price, the series editor, for his advice and inspiration; Andrew Nash, the copy-editor, for his help and patience; and finally my husband and family for spurring me on. I would also, of course, like to acknowledge the work of Roper, Logan and Tierney, which is the cornerstone of this book.

Charleen Newton

The author and publishers have made every effort to trace copyright holders, but if any have been overlooked the publishers will be pleased to make the necessary arrangements at the first opportunity. 